\title{
Architectural Skin color recognition algorithm based on moiré principle analysis
}

\author{
Rongke Jin $^{1, a}$, Yan Long ${ }^{2, b, *}$ \\ ${ }^{1}$ School of Engineering and Architecture, Jiaxing University, Jiaxing, Zhejiang, China \\ ${ }^{2}$ School of Urban Construction, Wuhan University of Science and Technology, Wuhan

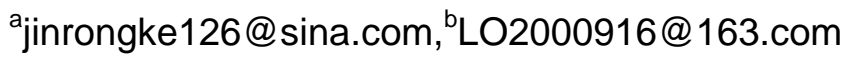

Keywords: Architecture skin color; Color features; Moiré principle.

\begin{abstract}
The traditional architecture skin color localization algorithm uses the gray image texture feature, the texture feature of gray image features and limitations bring great difficulty in getting an architecture skin color localization algorithm. In view of this, this paper puts forward the color of architecture skin color localization algorithm based on the moiré principle analysis. Firstly, two-dimensional wavelet decomposition for image, sliding windows is small ripple bedding features of images; And at the same time convert low-frequency sub-graph RGB color components as HSV color value, extract color feature, Second, wavelet variations positioning architecture skin color area; Finally to locate the architecture skin color positioning results of late correction processing and out-put. The algorithm adopts the comprehensive characteristics of localization, overcomes the drawback of using single feature localization and limitations, and can be seen from the results of this algorithm can accurately extract the architecture skin color location information from the background image.
\end{abstract}

\section{Introduction}

It has long been the self-evident fact that architecture has a close relation with its actual regional surroundings as well as the materials making it. In the era of pre-industrial times, nature had been the source of building material, so architecture is naturally matched with its regional surroundings. However, the great Industrial Revolution had brought along with steel, concrete and glass, which were the main building materials for the early Modernism Architecture. These architectures were later discarded due to its designing isolation to the surroundings. But the reflection and revision around this point has long been lasting until now. Therefore, it has gradually been the modern architect's cultural consensus to integrate surroundings into designing. Unfortunately, the current well-developed consumption culture and mass media have increasingly intensified a visually oriented thinking model on human, which has accordingly aroused a growing rush for architecture skin research, among which infatuation with Picture Collage, digitalization and pure visually centered materials are overwhelming. And the over-consideration on architecture skin has greatly broken in the dialogue between architecture and environment.

Architecture skin color Recognition is one of the critical parts in the Intelligent Architecture Systems and it occupies an important place in shriveling and manipulating the transportation. Vehicle architecture skin colors recognition system consists of three modules in general---architecture skin color location, character segmentation and character recognition, of which prompt and accurate location to the building plate is the prerequisite of ITS. At the same time, it is the critical technology in improving the recognition rates.

The accurate location and extraction of architecture skin colors is very important. So far the common methods of architecture skin color location include method based on color, based on gray-scale image and based on artificial neural network. In general, the method based on color will make the input RGB color image converted into a color image HIS, and then make the further location. However, when the color of the building plate area is close to the nearby color, usually the method based on the gray-scale image uses edge detection operator architecture skin color to produce vertical and diagonal texture, while the disadvantage is that the non-unify of the architecture skin 
color contrast under different lighting conditions will make the shapes and texture features not obvious, so it will influence the location results. The method based on artificial neural network need every extracting pixel feature inputted into neural network to study. As for the large amount of calculation, the architecture skin color location will take a long time, and deal with the local convergence as well.

According to this, we put forward the Architecture skin color Location Algorithm based on the Wavelet Texture Analysis. First of all, we pursue two-dimensional wavelet decomposition, calculating the wavelet texture feature within the sliding window image. And then we use the changes of the wavelet to locate a architecture skin color region. At last we check and manage the architecture skin color and output the positioning results. This algorithm adopts a comprehensive feature localization, so it overcomes the disadvantages and limitation of the single feature localization. From the results of the test we can see that this algorithm can extract the license plate location information from the background image. Here is the flowchart of the Color Architecture skin color Location Algorithm based on the Wavelet Texture Analysis.

\section{The wavelet analysis and texture feature of the architecture skin color}

The multi-resolution changes of wavelet can resolve the signal at any dimension into different frequency band. Now we take the color architecture skin color image as our subject investigated, use the Wavelet haar to two-stage decomposition, so as to decompose wavelet domain in the image to the sub graph signal which is in different spatial resolution, frequency characteristic, and feature. $\mathrm{T}$ represents the wavelet coefficients which is transformed once for a sub-band wavelet coefficients position(X, Y). A is taken from the set $\{\mathrm{LL}, \mathrm{LH}, \mathrm{HL}, \mathrm{HH}\}$; LL represents that horizontal and vertical directions are both low frequency; LH represents that horizontal direction is low frequency and vertical directions is high frequency; HL represents that horizontal direction is high frequency and vertical directions is low frequency; while $\mathrm{HH}$ represents that horizontal and vertical directions are both high frequency. Sub-frequency range- $\{\mathrm{LH}, \mathrm{HL}, \mathrm{HH}\}$ is increased from the edge of broad wise, endwise, and diagonal direction. The texture characteristic of architecture skin color location can be well reflected in high-frequency wavelet coefficient for the obvious edge characteristics in architecture skin color character regions, so it provides critical basis for architecture skin color location. At the same time, color information is obvious in low-frequency sub-graph, thus we can extract color characteristics. We consider the high frequency wavelet coefficients of calculation of statistical characteristics as texture features. These eigenvalues are average, mean square deviation, energy and inertia. We take texture features as the basis so as to definite the image regions. As for the window whose coverage region is $\mathrm{A}$ in the sub-band, the formula of the average, mean square deviation, energy and inertia in this region are as follows.

$$
\mu_{\theta}=\frac{1}{W \times H} \sum_{i=1}^{W} \sum_{j=1}^{H} I_{1}^{\theta}(\mu+i, v+j)
$$

\section{The HSV color features of architecture skin colors}

We transform the buildings image color of the background image wavelet low frequency spatially and then decompose it, namely transform space RGB(Red, Green, Blue) to space HSV(Hue, Saturation, Value), and extract color feature in the transformed color space. The method of transforming color space RGB to color space HSV is as follows:

$$
\sigma_{\theta}=\sqrt{\frac{1}{W \times H} \sum_{i=1}^{W} \sum_{j=1}^{H}\left[I_{1}^{\theta}(\mu+i, v+j)-\mu\right]^{2}}
$$

The basic idea of color extraction is: in the color space HSV, extract the color building image with component $\mathrm{H}$ and $\mathrm{S}$ and identify color region with component $\mathrm{V}$ subsidiarily, at the same time, characteristic the white region feature and black region feature with component $\mathrm{Z}$. Calculating the 
first and second moment of every component color's histogram so as to represent the distribution of the color in the image, and then extract the color feature.

Let $\mathrm{P}$ be the i-th color component of the i-th picture element in the picture, the first second and third component of the color in the region can be represented as: In this way, we can calculate and construct a color feature component in nine dimensional. And the specific form of this proper vector is as follows:

$$
E_{\theta}=\sum_{i=1}^{W} \sum_{j=1}^{H}\left[I_{1}^{\theta}(\mu+i, v+j)\right]^{2}
$$

\section{The Wavelet Transformed Architecture skin color Location:}

Calculate the lapse rate of every high frequency component chart decomposed by wavelet resolution. The reason why we calculate the lapse rate not the horizontal gradient is that the most obvious changes of the region building plate character is changes in lapse rate. Summing all the lapse rate and we can get intruding building plate region. The formula of summation of lapse rate is the picture that we sum the lapse rate of the three high frequency sub graph in alternative region, and then we use the summation feature of high frequency component lapse rate to resolve the region opponent of building license and building light in detail. Firstly let the lineage of picture be $\mathrm{m}$, column number be $\mathrm{n}$, and use a small rectangular whose lineage is $m$ and column number is 6 to search the whole picture from left to right, and write down the density value of every small rectangular region. (Density value is the total number of searched white pixels or picture elements in the small rectangular area). Secondly, calculating the density value in the whole picture as the threshold value. (The threshold value is the total number of white pixels or picture elements in whole picture). First of all, we characteristic the region whose threshold value is less than density value as 1 , the region whose threshold value is more than density value as 0 , and then scan these marks. The region marked 1 is obvious and region marked 0 is screening. As the result, we expand the region marked 1 to the left or to the right, and we can locate the left and right bound of building license.

\section{Searching for Alternative Building License Region}

The location of the building plate is by the side of the picture center. According to the situation, we put forward an architecture skin color region searching method from the center to around.

Identify the height and width of architecture skin color

Let the width of projected window be $\mathrm{W}$ and $\mathrm{H}$, which should be the same or bigger than the architecture skin color.

Let the sliding step of line be $1<\mathrm{w}<\mathrm{W}$, row be $1<\mathrm{h}<\mathrm{H}$, so we can identify the speed and precision.

The datum point of the projected window starts from the center of image.

Command line arguments increases $\mathrm{h}$, and line parameters increases $\mathrm{w}$

Command line arguments decreases $2 \mathrm{~h}$, line parameters decreases $2 \mathrm{w}$,

Command line arguments decreases $3 \mathrm{~h}$, line parameters decreases $3 \mathrm{w}$

Command line arguments decreases $4 \mathrm{~h}$, line parameters decreases $4 \mathrm{w}$

And so on.

In this way we can scan the data, so we can insure the definitely passing architecture skin color region in the scanning region, and improve the searching efficiency. As shown in the chart.

$$
G_{\theta}=\sum_{i=1}^{W} \sum_{j=1}^{H}(i-j)^{2} I_{1}^{\theta}(\mu+i, v+j)
$$

\section{Subsequent Treatment of the Architecture skin color Region}

Next, an important work we should do is that the correction for grade after the location, whose quality will affect the subsequent identify of architecture skin color character. Firstly, we identify the angle of inclination, and then take projection on the direction of the angle, so we can get the sole, and then 
identify the boundary of the plate character. The node shift of the plate is within plus or minus 15 degrees. According to this, we can get the angle of the plate through calculation. The method is as follows.

1. Start from the architecture skin color in the horizontal direction, and take 3 degree as the step length, and then project it along the counter clockwise and the clockwise.

2. Statistic the black picture element at every line after the projection. And then pick out the changes gradient of the 3 adjacent rows of black pixels from the result of statistics. Keep the greatest difference of the result.

\section{The experiment result}

The building plate picture we adopt is mainly taken by digital camera at random under natural condition, whose size is $640 * 480$. The building plate region accounts for $3 \%-10 \%$ in the whole building plate picture. Also there are some pictures---the building plate pictures of headstock or tailstock of a building, which are snapped by a camera. We simulate and verify the architecture skin color picture of the architecture skin color location arithmetic in this thesis in all kinds of lighting conditions and backgrounds. The digital pictures are shown in the fig1 and fig 2.
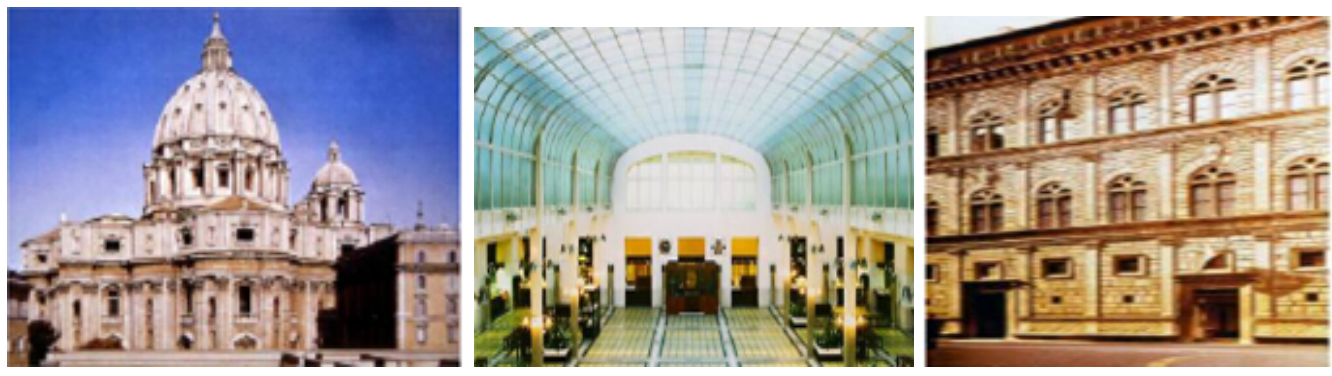

Fig 1 the original picture
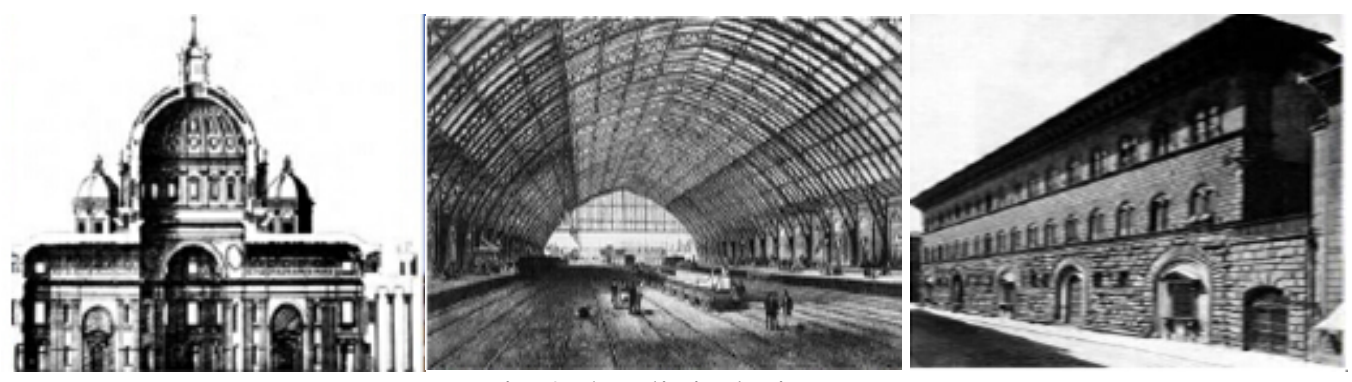

Fig 2 the digital picture

We identify the following evaluation parameters in order to judge the experiment.

The correct detection rate: the proportion of correctly detected building plates' number and the total number of building plates in detecting set.

The false detection rate: the proportion of false building plate and total number of building plates in detecting set, including the condition that a building does not have building plate and we detect the building plate.

The missing detection rate: the proportion of building plates we are not detected and the total number of building plates in detecting set.

The experiment data is as follows:

$$
T=\left(\mu_{L H}, \sigma_{L H}, E_{L H}, G_{L H}, \mu_{H L}, \sigma_{H L}, E_{H L}, G_{H L}, \mu_{H H}, \sigma_{H H}, E_{H H}, G_{H H}\right)^{T}
$$

The analysis of the experiment results

1. The texture and color feature have different location effect under diverse weight assemble, so is the speed. The bigger proportion which color feature accounts for weight, the less time. The best weight of color feature is 0.6 , and the bigger weight will make the recognition rate decreased. 
2. The accuracy rate of this method in the thesis is better than the other three calculation I have tested, however, the location speed is in inferiority.

3. This method uses comprehensive feature to locate architecture skin color, which overcomes the disadvantage and limitation of single feature location, at the same time, it is less effected by the texture and lighting condition of a building, and so on.

Experiment has testified that the method in this thesis is very good to deal with the building license location. At the same time, it provides the theoretical and significance application of value to wavelet texture analysis technology, extraction of feature and matching technology.

\section{Conclusion}

In this thesis, we mainly introduced the color architecture skin color location algorithm based on wavelet texture analysis. Firstly, we take a two dimensional wavelet decomposition to the picture, calculating the wavelet texture feature of the picture in the projected window, then transform the low frequency to the RGB color component and HSV chromatic value at the same time, extracting the color feature and get the comprehensive feature vector. Secondly, we use the wavelet changes to locate the architecture skin color region. Finally, we post-process the building plate. This method uses comprehensive feature to locate architecture skin color, which overcomes the disadvantage and limitation of single feature location, so it can avoid the mixing randomized texture noise in buildings picture. The experiment has testified that the building picture can have good texture feature extraction effect in complex lighting condition and texture. So it improves the accuracy rate of architecture skin color in large extent.

\section{References}

[1]Gao Q,Wang X N,Xie G F. Architecture skin color Recognition Based On Prior Knowledge[A].Proceedings of the IEEE International Conference on Automation and Logistics. 2007:2964-2968.

[2]Keerthi S S,Lin C J.A symptotic behaviors of support vector machines with Gaussian kernel[J]. Neural Computation.15July2003:1667-1689.

[3]Chen Z X, Liu C Y. A Novel Algorithm of Architecture skin colors Automatic Location Based on Texture Feature [A]. IEEE International Conference on Automation and Logistics. 2009, 5. pp: 1360-1363.

[4]Kaushik Deb,Kang-Hyun Jo. HSI Color based building Plate Detection [A].International Conference on Control, Automation and Systems.2008, 14-17:687-691.

[5] David Leather barrow. The Roots of Architectural Invention: Site, Enclosure, Materials New York: Cambridge University Press.1993.

[6] Xiang biao Zhao .Global Architecture now. Scientific\& Cultural Publishing.2008. 